\title{
The warping of extra spaces accelerates the expansion of the universe*
}

\author{
Ishwaree P. Neupane ${ }^{\dagger}$ \\ Department of Physics and Astronomy, University of Canterbury \\ Private Bag 4800, 8041 Christchurch, New Zealand
}

March 31, 2010

\begin{abstract}
Generic cosmological models derived from higher dimensional theories with warped extra dimensions have a nonzero cosmological constant-like term induced on the $3+1$ space-time, or a physical 3-brane. In the scenario where this $3+1$ space-time is an inflating de Sitter "brane" embedded in a higher-dimensional space-time, described by warped geometry, the $4 \mathrm{D}$ cosmological term is determined in terms of two length scales: one is a scale associated with the size of extra dimension(s) and the other is a scale associated with the warping of extra space(s). The existence of this term in four dimensions provides a tantalizing possibility of explaining the observed accelerating expansion of the universe from fundamental theories of gravity, e.g. string theory.
\end{abstract}

\footnotetext{
*Essay written for the Gravity Research Foundation 2010 Awards for Essays on Gravitation; Selected for an "Honorable mention" 15 May 2010.

†E-mail: ishwaree.neupane@canterbury.ac.nz
} 
Physicists today are faced with a number of mysteries about the universe which have called for elegant and straightforward explanations. One major mystery uncovered nearly 12 years ago from observations of distant supernovae [1] is that the expansion of the present-day universe is accelerating rather than slowing down.

In the standard model of cosmology, first, we introduce a cosmological constant $\Lambda$, which has the same effect as an intrinsic energy density of the vacuum (and an associated negative pressure). It is used to balance the total energy budget and explain the accelerating expansion observed in the more recent history of our universe. Second, we put in dark matter, which plays a key role in the formation of cosmic structure. Third, we demand the occurrence of cosmic inflation at any early epoch - a period of exponential expansion that explains why the universe is smooth on the largest scales.

The Einstein's cosmological constant appears to be the most parsimonious solution to a late time cosmic acceleration [2]. Yet, a canonical picture of the standard model of cosmology as above raises some conceptual issues, such as, what is the origin of a cosmological term in Einstein's theory of general relativity (GR)? Is gravity purely geometrical as Einstein thought, or is there more to it (such as, extra dimensions, scalar fields)? What made our universe so big? This last question can be addressed by invoking a period of cosmic inflation, but again we do not know the physical origin of gravitationally repulsive energy driving inflation. Also, there is no known natural way to derive a tiny cosmological constant - that the observations favour - from fundamental theories of gravity.

Given that the physics in higher spacetime dimensions defines a framework of lowenergy four-dimensional field theory and a unique answer to the gravitational vacuum energy density in today's universe, there can be a unique prediction for the cosmological constant. I want to argue, in this essay, that the cosmological constant of our spacetime may well be determined by two parameters: one is a scale related to the size of extra dimensions and the other is a scale related to the warping of extra dimension(s). This essay, building on some new ideas, provides a key conceptual consolidation.

Physicists have long sought a theory of quantum gravity that would unify all the principles of physics in a pleasing mathematical formalism. The history of 20th century physics has itself been a struggle to find a way to unite general relativity and quantum mechanics. One of the ways in which physicists have tried to unify gravity with the other forces is to create theories that allow for many more spatial dimensions. A serious motivation for considering additional dimensions came from string theory, which in turn is inspired by the failure of classical (Einstein) gravity to work at very short distance scales.

In the early 1980s, certain ten- and eleven-dimensional supergraviy models - in the low energy limits of 10D string theory and 11D M-theory - were realized as profound theoretical tools in connecting quantum mechanics with general relativity. The major achievement in the mid 1990s was a series of new understandings about the role of localized sources such as 'branes' and fluxes [3]. These ingredients in string/M-theory have helped us to learn several new ideas in physics, including the localization of gravity on a brane embedded in a warped five-dimensional anti-de Sitter space [4], gravity - gauge theory dualities [5] and methods of constructing de Sitter vacua in string theory [6]. 
Despite some novelties, it is not straightforward to explain cosmic acceleration directly through a standard compactification of ten- or eleven-dimensional supergravity models. There is a 'no-go' theorem, due to Gibbons [7], Maldacena and Nunez [8] and many others [9], which basically asserts that if we compactify any string-derived supergravity model on a smooth compact manifold, then it would be difficult to obtain an inflationary cosmology as a background solution of 10D or 11D classical supergravities.

Since the universe appeared to be both past and future de Sitter (albeit with vastly differing vacuum energies) this would seem to be a problem. In turn, in recent years attempts have been made around this particular no-go result. The original no-go theorem assumes time independence of the internal space, and so one could look for timedependent solutions. Following this intuition, the author and many others [10, 11] have constructed varieties of time-dependent compactifications which describe an accelerating universe. In several examples studied in the literature, with maximally symmetric extra dimensions, solutions to D-dimensional Einstein equations (with $D \geq 5$ ) only support a transiently accelerating universe with time-dependent metric moduli.

Indeed, almost all solutions found using time-dependent metric moduli have the property that as one moves to the minimum of the potential the extra dimensions grow or shrink slowly or even stabilise in a few specific cases [12], resulting into an exponential type potential. One can now imagine "bouncing" the universe off this potential. Albeit for a brief interval, the energy is dominated by the potential term of metric moduli, or scalar fields, and the universe undergoes a transient period of cosmic acceleration [11].

To that end, we find interest in an alternative scenario where the extra dimensions are warped but time-independent. One of such proposals is the five-dimensional 'warped' braneworld model proposed by Randall and Sundrum [4]. Their proposal raises the possibility that at least one of the extra dimensions postulated by string theory could be large enough to have experimental implications, including that in particle physics.

If we learned one thing from higher dimensional theories of gravity, including string theory, it is that extra dimensions play major tricks in a spacetime that is dynamical. In what follows, I use very little of the full formalism that has been developed to describe string theory, rather motivate the readers to one basic fact. Gravity, Einstein asserted, is caused by a warping of space and time - or, in a language many physicists prefer, by a warping of spacetime. Although Einstein's original assertion was based on dynamics in a stationary and in a four-dimensional spacetime, the same is true in a dynamical background and with an arbitrary number of spacetime dimensions.

One quite simple, but equally robust, observation is that, in spacetime dimensions $D \geq 5$, with $(D-4)$ warped extra dimensions, a four-dimensional observer would measure a nonzero positive cosmological constant, which is induced not by a D-dimensional cosmological term but by the curvature related to the expansion of a physical $3+1$ spacetime. This basic idea can be illustrated with a five-dimensional 'warped metric', maintaining the usual four-dimensional Poincaré symmetry,

$$
d s_{5}^{2}=e^{2 A(y)}\left(\hat{g}_{\mu \nu} d x^{\mu} d x^{\nu}+\rho^{2} d z^{2}\right), \quad A(z)=-\mu|z|,
$$


where $x^{\mu}$ are the usual coordinates $(\mu, \nu=0,1,2,3), \rho$ is a length scale associated with the size of extra space, $e^{A(y)} \equiv e^{-\mu|z|}$ is the warp factor as a function of the fifth dimension $z$. Indeed, the problem of the vacuum energy density or cosmological constant - why it is extremely small by particle physics standard - arises only in a dynamical spacetime. What it means is that the usual 4D line element must be time-dependent, such as

$$
\hat{g}_{\mu \nu} d x^{\mu} d x^{\nu}=-d t^{2}+a^{2}(t)\left[\frac{d r^{2}}{1-k r^{2}}+r^{2}\left(d \theta^{2}+\sin ^{2} \theta d \phi^{2}\right)\right],
$$

where the curvature constant $k=0,+1$ and -1 , respectively, for flat, closed and open universes. With (2), the metric (1) becomes an exact solution of 5D Einstein equations, following from

$$
S \propto \int d^{5} x \sqrt{-g_{5}} R_{5}
$$

when the scale factor of the universe $a(t)$ takes the form

$$
a(t)=\frac{1}{2} \exp \left(\frac{\mu t}{\rho}\right)+\frac{k \rho^{2}}{2 \mu^{2}} \exp \left(-\frac{\mu t}{\rho}\right),
$$

where $\mu$ is some constant (with inverse length dimension). When $\mu=0$, it is mandatory to take $k=0$ and also introduce a 5D cosmological term, or a source in the 5D bulk spacetime, which corresponds to a static configuration studied in Ref. [4].

Especially, in the $k=-1$ case, there is a big-bang type singularity at

$$
t=\frac{\rho}{2 \mu} \ln \frac{\mu^{2}}{\rho^{2}},
$$

whereas in closed and spatially flat universes, the scale factor is nonzero. This is desirable because the generic singularity of time-dependent solutions of general relativity is generally unacceptable - as it cannot have any quantum interpretation. More remarkable is the fact that the universe naturally inflates when $\mu>0$ or the warp factor is not constant.

In the discussion above, I assumed for simplicity that the 5D bulk cosmological term is zero, but a similar solution exists when one modifies the $5 \mathrm{D}$ classical action as

$$
S \propto \int d^{5} x \sqrt{-g_{5}}\left(R_{5}-2 \Lambda_{5}\right)
$$

The only difference now is that the warp factor is given by

$$
A(z)=\ln \left(24 \mu^{2}\right)-\ln B(z), \quad \text { where } \quad B(z)=24 \mu^{2} e^{\mu|z|}+\Lambda_{5} \rho^{2} e^{-\mu|z|},
$$

which reduces to the earlier result when $\Lambda_{5}$ is set to zero. In the above I replaced $z$ by $\bmod z$, or imposed a $\mathcal{Z}_{2}$ symmetry at $z=0$; the reason was that in a physical scenario, one has to solve the D-dimensional Einstein equations not just for $|z|>0$ but also at the 
position of a physical 3-brane or the $4 \mathrm{D}$ hypersurface at $|z|=0$, which can in principle have a nonzero 3 -brane tension $T_{3}$, described by the action

$$
S=\frac{M_{5}^{3}}{2} \int d^{5} x \sqrt{-g_{5}}\left(R_{5}-2 \Lambda_{5}\right)+\int d^{4} x \sqrt{-g_{b}}\left(-T_{3}\right),
$$

where $M_{5}$ is the 5D Planck mass. The 5D Einstein equations

$$
G_{A B}=-\Lambda_{5} g_{A B}-\frac{T_{3}}{M_{5}^{3}} \frac{\sqrt{-g_{b}}}{-g_{5}} g_{\mu \nu}^{b} \delta_{A}^{\mu} \delta_{B}^{\nu} \delta(z),
$$

following from the action (8), are explicitly solved when

$$
T_{3}=\frac{6 \mu M_{5}^{3}}{\rho^{2}}\left(1-\frac{\Lambda_{5} \rho^{2}}{24}\right) .
$$

It is assumed that $\Lambda_{5}<0$ or $\Lambda_{5}=0$, which both lead to a positive brane tension. In the $\Lambda_{5}>0$ case we can actually relax the $Z_{2}$ symmetry, which still leads to a finite $4 \mathrm{D}$ Newton constant, but we then need to satisfy $\Lambda_{5}<24 / \rho^{2}$ so as to get a positive brane tension.

An important observation here is that the brane tension is induced by the curvature related to the expansion of $3+1$ spacetime alone, which is determined by the slope of the warp factor. In a wide class of warped compactifications, which include string theory, this suggestion has startling implications.

First, the physical universe inflates naturally when the warping of extra space is nonzero. This happens because the warping of extra space(s) generates in the four-dimensional effective theory a cosmological constant-like term $\Lambda_{4}$. To show this, we focus here on the $R_{5}-2 \Lambda_{5}$ term from which we derive the scale of gravitational interactions:

$$
\begin{aligned}
S_{\mathrm{eff}} & \supset \frac{M_{5}^{3} \rho}{2} \int e^{3 A(z)} d z \int d^{4} x \sqrt{-\hat{g}_{4}}\left(\hat{R}_{4}-\frac{12}{\rho^{2}}\left(\partial_{z} A\right)^{2}-\frac{8}{\rho^{2}} \partial_{z}^{2} A-2 \Lambda_{5} e^{2 A(z)}\right) \\
& \supset \frac{M_{5}^{3} \rho}{2} \int\left(\frac{24 \mu^{2}}{B(z)}\right)^{3} d z \int d^{4} x \sqrt{-\hat{g}_{4}}\left(\hat{R}_{4}-\frac{12 \mu^{2}}{\rho^{2}}+\frac{768 \Lambda_{5} \mu^{4}}{B^{2}(z)}\right) .
\end{aligned}
$$

When $\Lambda_{5}=0, \Lambda_{4}\left(\equiv 6 \mu^{2} / \rho^{2}\right)$ is constant, whereas, with $\left|\Lambda_{5}\right|>0$, it is position dependent.

Second, as advertised above, the vacuum energy density is uniquely determined in terms of two length scales: in the $\Lambda_{5}=0$ case, the two physically relevant parameters are $\mu$ and $\rho$, whereas, in the $\left|\Lambda_{5}\right|>0$ case, by defining $\Lambda_{5} \equiv-6 / \ell^{2}$, we can see that, other than the $\mu$, the ratio $\rho / \ell$ is more relevant (for determining $\Lambda_{4}$ ) than $\rho$ or $\ell$ separately.

Third, from Eq. (11), we find that the relation between four- and five-dimensional effective Planck masses is given by

$$
M_{P l}^{2}=M_{5}^{3} \rho \int_{-\infty}^{\infty} d z \frac{1}{\left(e^{\mu|z|}-c^{2} e^{-\mu|z|}\right)^{3}} \approx \frac{M_{5}^{3} \rho}{4 \mu c^{2}}\left(\frac{1+c^{2}}{\left(1-c^{2}\right)^{2}}-\frac{\ln \left(1-c^{2}\right)}{2 c}\right),
$$


where $c^{2} \equiv\left(-\Lambda_{5}\right)\left(\rho^{2} / 24 \mu^{2}\right)$. In the limit $\mu \rightarrow 0$, a new dimension of spacetime opens up, since $M_{4}^{2} \rightarrow \infty$, but with a nonzero $\mu$ (and a finite $c$ ) the $4 \mathrm{D}$ effective Newton's constant is finite. All the cosmological features described above extend far beyond 5D theory.

One basic reason for extending the discussion above to ten dimensions [13], or more specifically, string theory, is that it includes generalizations of both standard quantum field theory and 4D Einstein's theory. If "spacetime" is an emergent phenomenon, as suspected by many string theorists, then some of the extra dimensions predicted by string theory can be realized only when we probe sufficiently small distances. From this viewpoint, it would be reasonable to assume that five of the extra dimensions (predicted by string theory) are much smaller in size than the radial (fifth) dimension. A few explicit examples were provided in the Refs. [13, 14], where explicit solutions describing an accelerating expansion of the universe were found just by solving the 10D Einstein equations.

I conclude with a few remarks.

One of the most exciting developments in cosmology is the suggestion that the warping of extra dimensions plays a key role in determining the magnitude of the four-dimensional cosmological constant and hence the rate of expansion of our physical universe.

The key point in the present method of explaining an accelerating expansion of the universe is the treatment of 'brane' as a four-dimensional physical spacetime and consideration of a non-factorizable (warped) background geometry in higher dimensions. Within this set-up, the existence of an effective cosmological constant-like term in four dimensions provides a tantalizing possibility of explaining an accelerating expansion of the universe from fundamental theories of gravity, which include string theory.

String theory as it is currently formulated and interpreted has a huge number of equally plausible solutions, called string vacua [6]. These vacua might be sufficiently diverse to explain various phenomena we might observe at lower energies. If these properties are true, string theory as a theory of quantum gravity would have little predictive power for low energy particle physics experiments and also for cosmologies. Because the theory is difficult to test, some theoretical physicists have asked if it can even be called a scientific theory of everything. In the book The Trouble with Physics, Lee Smolin takes a complex debate on topics such as string landscape and ability of string compactificatons to explain the cosmological constant problem. But, it is difficult to imagine that a completely wrong theory could generate so many good ideas, including microscopic descriptions of inflation from D-braneworld models and dualities between closed string theories that contain gravity and decoupled open string theories that don't.

Most importantly, we are not arguing that string theorists' inability to empirically test their results in a cosmic lab will disappear soon; shifting the emphasis from conventional explanation to cosmic acceleration and/or cosmological constant problem to a boarder picture of D-dimensional Einstein's theory, with one or more warped extra dimensions, leads to a general class of accelerating cosmologies which include in four dimensions Einstein's gravity supplemented by an effective cosmological constant-like term.

In the coming years we all hope to learn much more about inflation and observed 
cosmic acceleration of the universe (attributed to a small positive cosmological constant or other fluid-like "dark energy") from the highly refined computations and sophisticated observations. More data from precision cosmology and large hadron collider at CERN might help to address questions about the early universe and the high energy frontiers. The years ahead will certainly bring even more twists, breakthroughs and surprises in gravity and cosmology research.

\section{Acknowledgement}

This work was supported by the Marsden fund of the Royal Society of New Zealand.

\section{References}

[1] A. G. Riess et al. Astron. J. 116, 1009 (1998); S. Perlmutter et al., Astrophys. J. 517, 565 (1999).

[2] WMAP Project Webpage [http://map.gsfc.nasa.gov].

[3] J. Polchinski, Phys. Rev. Lett. 75, 4724 (1995).

[4] L. Randall and R. Sundrum, Phys. Rev. Lett. 83, 3370 (1999); L. Randall and R. Sundrum, Phys. Rev. Lett. 83, 4690 (1999).

[5] J. Maldacena, Adv. Theor. Math. Phys. 2, 231 (1998).

[6] S. Kachru, R. Kallosh, A. Linde and S. P. Trivedi, Phys. Rev. D 68, 046005 (2003).

[7] G. W. Gibbons, in Supersymmetry, Supergravity and Related Topics, edited by F. del Aguila, J. A. de Azcarraga, and L. E. Ibanz (World Scientific, 1985), pp. 123-146.

[8] J. M. Maldacena and C. Nunez, Int. J. Mod. Phys. A 16, 822 (2001).

[9] S. B. Giddings, S. Kachru and J. Polchinski, Phys. Rev. D 66, 106006 (2002).

[10] P. K. Townsend and M. N. R. Wohlfarth, Phys. Rev. Lett. 91, 061302 (2003); N. Ohta, Phys. Rev. Lett. 91, 061303 (2003).

[11] I. P. Neupane, Class. Quant. Grav. 21, 4383 (2004); I. P. Neupane and D. L. Wiltshire, Phys. Rev. D 72, 083509 (2005).

[12] I. P. Neupane, Phys. Rev. Lett. 98, 061301 (2007).

[13] I. P. Neupane, Class. Quant. Grav. 26, 195008 (2009); Class. Quant. Grav. 27, 045011 (2010);

[14] I. P. Neupane, Phys. Lett. B683, 88 (2010). 